\title{
Flexible job control in heterogeneous production structures
}

\author{
D. Ansorge, C. Effert \\ Technische Universität München \\ Institute for Machine Tools and Industrial Management (iwb) \\ Boltzmannstrasse 15, 85748 Garching, Germany \\ e-mail: dirk.ansorge@iwb.tum.de,christian.effert@iwb.tum.de
}

\begin{abstract}
The existing basic approaches of production planning and control permit an adaptation of control strategies to changing production systems only to a limited degree. However, adaptations are necessary because of the prevailing turbulence in the market and production environment.

This paper presents the concept of "negotiation-based production coordination", an approach to scale the order processing control between central and decentralized by means of various degrees of planning scope. By monitoring the logistic parameters and with the aid of event-discrete simulation, the need for a change in strategy change can be recognized and the changes implemented.
\end{abstract}

\section{Keywords}

production planning and control, decentralized planning, negotiation, coordination

\section{INTRODUCTION}

The adaptation of production to numerous product variants, the concentration on specific manufacturing technologies, the immobility of machines and the increasing trend towards decentralization lead to heterogeneous production structures. This means the simultaneous existence of different organizational structures, for example workshop manufacturing and group technology.

This situation imposes high requirements on order processing, because work cannot proceed with only one production planning and control strategy. Depending on product, order category, general technical conditions and the required processing time, different strategies and their adaptation to the current situation are necessary. For this purpose, conventional central or purely decentralized approaches to production planning and control are not suitable. In particular scaling is not possible between central and decentralized control. 
An important precondition for adaptation of the production planning and control strategy is that the structure and workflow organization in manufacturing should basically permit both central and decentralized control of order processing. The iwb has therefore developed the concept of negotiation-based production coordination, within the project FLEXIFEIN funded by the German Bundesministerium für Bildung und Forschung $(\mathrm{BMB}+\mathrm{F})$. The concept considers the requirements and makes it possible to determine methodically suitable planning and control strategies and implement them in production.

\section{CONCEPT OF NEGOTIATION-BASED PRODUCTION COORDINATION}

The concept of negotiation-based production coordination presupposes a structuring of production into autonomous production areas (see figure 1). Their capabilities can be made available to production as services. They are able to plan, carry out and control tasks on their own.

The orientation of job control towards global objectives as well demands in addition a central instance superior to production with a product-oriented view of production orders, in other words a coordinator (see figure 1). He forms the interface both to the pre-production and the autonomous production areas. Moreover, he creates positive general conditions (e. g. clear order situation, procurement of time-critical material) for the order processing in the autonomous production areas and intervenes in the case of conflicts that cannot be solved locally.

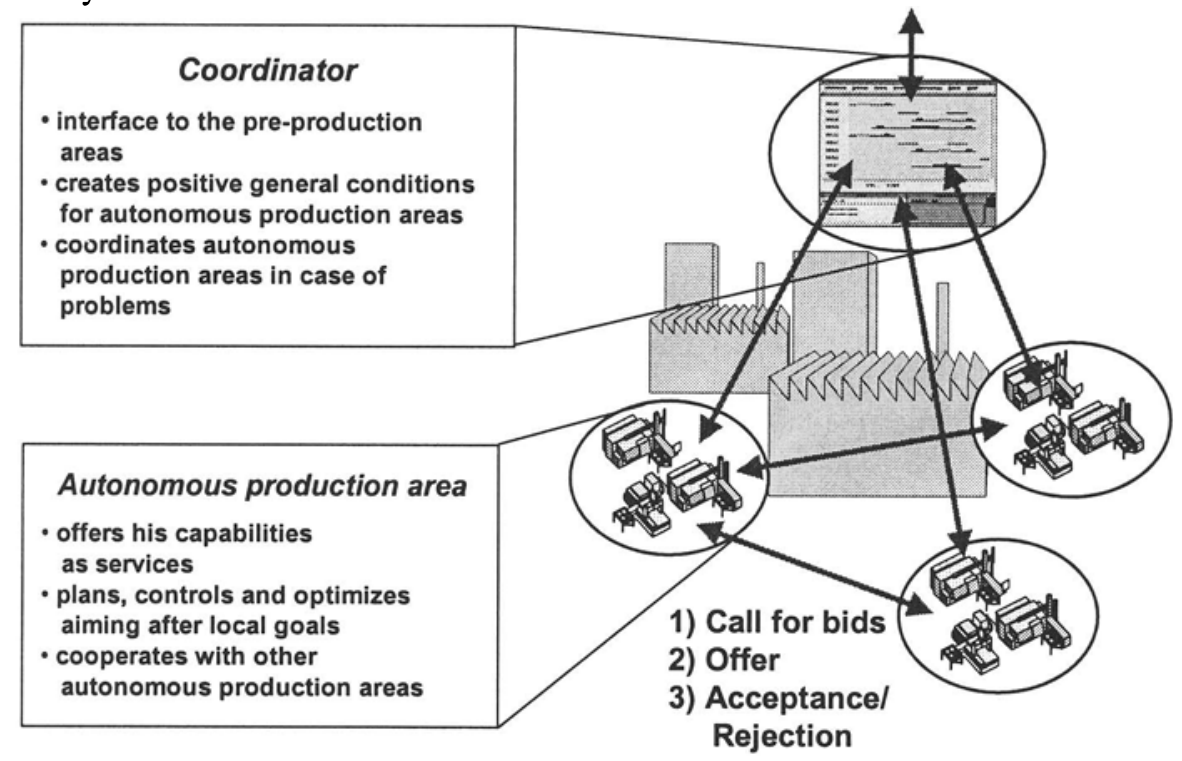

Figure 1 Production coordination 
For the allocation of the production orders and/or parts of the production orders to autonomous areas by the coordinator as well as for their cooperation among one another, a uniform communication mechanism has been developed. With "call for bids" and "offer" as its elements, it corresponds to the market principle of negotiation. The communication mechanism, which can easily be automated and standardized, permits equal treatment of different internal and external service providers.

\section{VARIATION OF DEGREE OF DECENTRALIZATION WITHIN ORDER PROCESSING}

As stated initially, control of order processing requires individual adaptation depending on production structure, order type (e.g. serial or individual order) and order situation. In particular, scaling between central and decentralized control must be possible. Serial orders can be produced in suitable autonomous areas with high local responsibility. Unusual or new products, on the other hand, require a large amount of central support. The scaling of order control between central and decentralized is achievable by

- precise variation of the scope available for planning and decision and

- precise variation of the method of allocating orders, from disposing to coordinating.

\subsection{Variation of the scope for planning and decision}

The coordinator as well as the autonomous production areas can plan tasks with different planning scopes. Planning scopes are defined as the variation in the task volume, variation of the time available for execution of a task and variation in the allocation of resources.

In this way, planning can be varied from precise time scheduling of operations without scope for modification of the available time up to approximate orderrelated planning with scope available for such modifications, depending on the situation. In this case a specific resource, a resource group or merely a capability can be assigned to a task.

The scope for decision available to an autonomous production area refers on the one hand to the ability to decide on the acceptance of a task itself, and on the other hand to the ability to transfer a task already accepted or parts of it to another autonomous production area. This scope is particularly necessary for the decentralized order processing.

An essential base for the mapping of the scope in each case is the design of the equivalent data model. In the data model applied here, the degrees of freedom can be represented by parameter sizes adjustable continuously and in discrete form. 


\subsection{Variation in the form of task allocation}

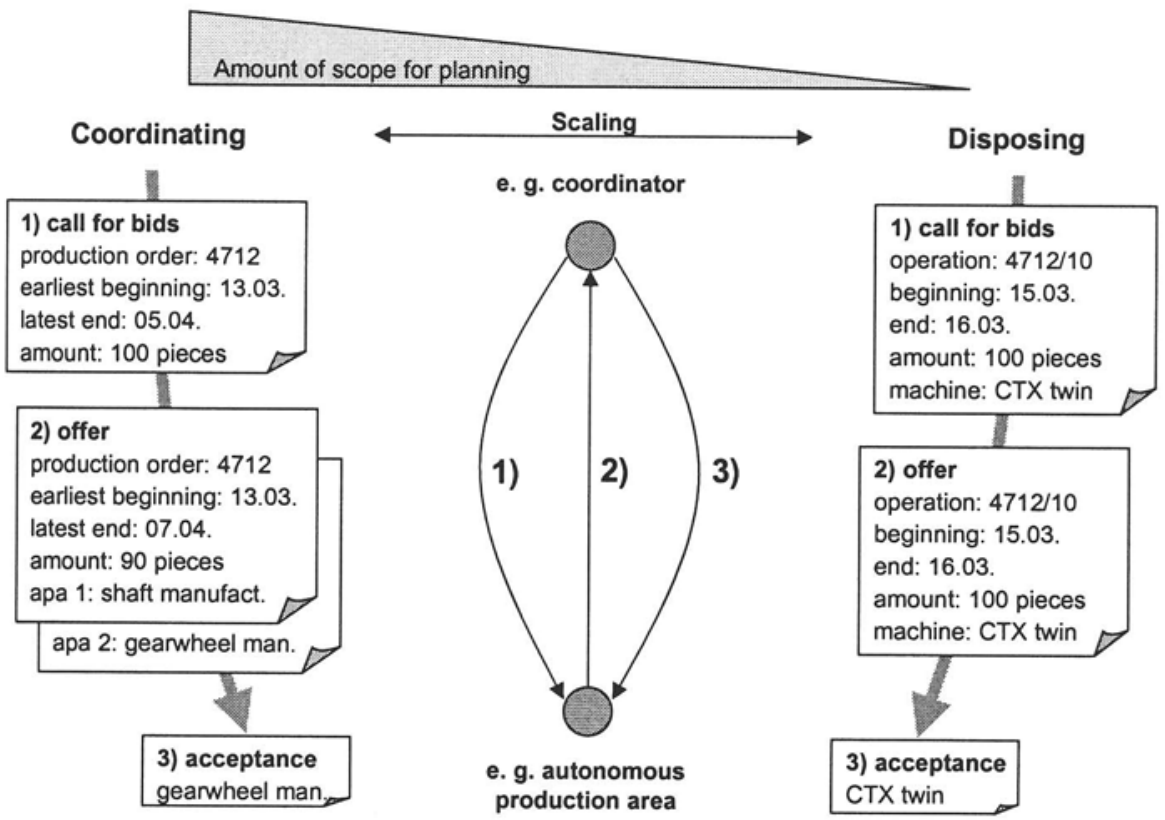

Figure 2 The coordinating and disposing processes in order allocation

While the central order processing provides for "disposing" task distribution, means for the coordination between autonomous production areas (deadlines, capacities) must be created for the decentralized order processing. A communication mechanism which supports both methods of placing orders is therefore necessary. The negotiation mechanism already mentioned has proved to satisfy this requirement. By the "call for bids", "offer" and "offer selection" principle, many opportunities for coordination between the negotiation partners can be created. On the other hand tasks can also be allocated to resources by restrictive conditions for calls (see figure 2).

\section{CHANGES IN CONTROL STRATEGIES}

A precondition for a precise change in control strategies is monitoring of the autonomous production areas with regard to the aims that have been determined. Important production-logistic aims here are adherence to schedules, processing time, stock levels and quality (see figure 3). Quality is described by the proportion of parts without failures produced by an autonomous production area. The aims are determined according to the previously defined order types. If the observed aims deviate from the reference values that are to be achieved, a change in the actual 


\section{Flexible job control in heterogeneous production structures}

strategy has to be carried out by adjusting the degrees of freedom, taking current operating state into consideration.

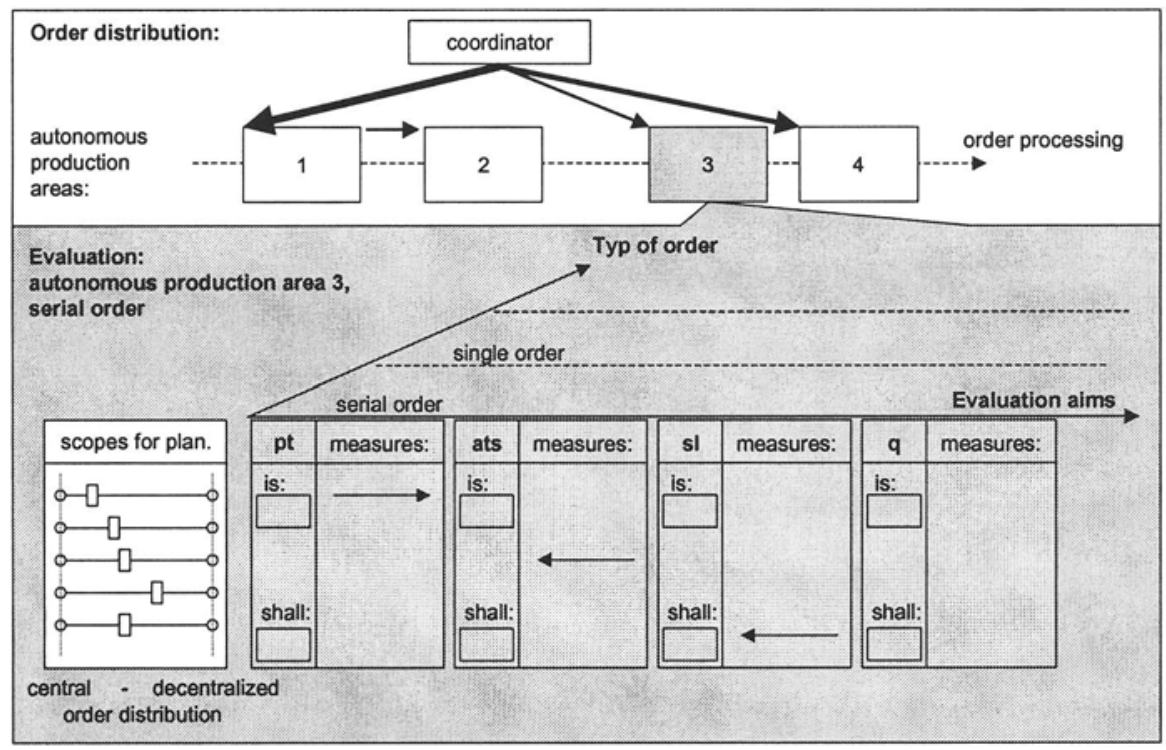

Figure 3 Monitoring of autonomous production areas, e. g. area 3

For this purpose, a knowledge base is used to store the ways in which specific regulation of planning scope takes effect on the aims, depending on order types. In the first step the variety of possible parameter combinations (scopes for planning and decision, types of task allocation, see figure 3 ) is reduced by restriction to reasonable combinations. Those combinations that cause no contradiction between central and decentralized control strategy from a logistic and organizational point of view are designated a priori as reasonable. This means for example, that within a planning hierarchy no variation between central and decentralized control should occur. Changes to scope parameters that cause a substantial or longer-term change of workflow organization in the autonomous production areas must not be executed without an arrangement with the employees, for industrial-psychology reasons. Scopes for planning which, on the other hand, have no effect on the existing organization, for example those related to scheduling, are automated in the data processing and adapted to the given situation.

\section{VERIFICATION OF A PARAMETER SET}

An evaluation as to whether the desired aims can be achieved with the degrees of freedom adjusted again is undertaken with the aid of event-discrete simulation. The simulation model describes the capabilities of the autonomous production areas, their task-related behavior and their communications interface. Task-related 
behavior includes behavior relating to processing time and adherence to schedules in accordance to the task volume. From experience, a production order which is processed completely in a group technology environment, for example, has a shorter processing time than tasks with individual jobs carried out in different workshops. In this way, behavior relating to processing time and adherence to schedules improves as more jobs are dealt with at a process-oriented autonomous production area. Since the current workload situation is important for the behavior description, too, operating characteristics according to Wiendahl (1997) are used as description model.

Using a data processing link between the coordinator and the simulation model, different scenarios are simulated and evaluated according to aspects of production logistic. For this purpose, a limited-lot production was adopted for the model. The simulation runs yielded comparable results concerning the logistic aims. After this the degrees of freedom were changed with system support. For some production areas, an improvement in the order processing could be achieved by decentralization. In other, workshop-oriented production areas which were affected by intense variations of the task spectrum, it was necessary to maintain central overall planning, though deadline adjustment with low-load areas was able to take place on a purely decentralized basis.

The simulation experiments showed that frequent changes of planning scope lead to an uncontrolled oscillation effect in the overall system. In order to avoid this, interaction with the persons involved in order planning and control is indispensable if a change in scope is to take place.

The data processing feasibility of the concept was demonstrated on the basis of component based client/server software architecture at the iwb as a constituent of the model factory.

\section{CONCLUSION}

Based on the concept of negotiation-based production coordination, a method has been presented which supports adaptation of the order processing to changing production and order situations by means of scalable scope. The scopes concern, on the one hand, the planning of order processing (task volume, time available for execution, resources) and the decision about the acceptance of a task and/or its partial or complete forwarding to another autonomous production area. On the other hand, the form of task allocation can be varied between disposing and coordinating. A communication mechanism based on the market principle of negotiation is used for adjusting the autonomous production areas.

Essential constituents of the method are the monitoring of selected productionlogistic aims and simulation-supported derivation of measures. The monitored aims are determined depending on the task types defined before. A knowledge base is used to store the ways in which specific regulation of planning scope takes effect on these aims. The adjusted scenarios are inspected with the aid of event-discrete 
simulation, to decide whether the desired aims can be achieved by the intended change of the degrees of freedom.

\section{REFERENCES}

Ansorge, D. and Koller, A. (1996) Intelligent decentralized planning and complex strategies for negotiation in flexible manufacturing environments, in Robotics and manufacturing - recent trends in research and applications (ed. Jamshidi), Proceedings of the sixth international symposium on Robotics and manufacturing (ISRAM '96), ASME Press, New York.

Hahndel, S. and Levi, P. (1994) A distributed task planning method for autonomous agents in a FMS. IROS '94 - IEEE/RS/GI Intelligent Robots and Systems, Munich, 1285-92.

Pischeltsrieder, K. (1996) Steuerung autonomer mobiler Roboter in der Produktion. Springer Publishing, Berlin et al.

Reinhart, G. and Ansorge, D. (1997) Beherrschung flexibler Abläufe durch dezentrale Leittechnik. ZWF CIM, 10, 514-7.

Smith, R. G. (1980) The contract net protocol: high level communication and control in a distributed problem solver. IEEE Transaction on computers, C-29, 12.

Wiendahl, H.-P. (1997) Betriebsorganisation für Ingenieure. Carl Hanser Publishing, Munich et al.

\section{BIOGRAPHY}

Dirk Ansorge was born in Munich, Germany, in 1968. From 1989 till 1994 he studied mechanical engineering at the Technische Universitaet Muenchen. Since then he works as an assistant at the Institute for Machine Tools and Industrial Management $(i w b)$. His research interests include logistics, production planning and control as well as simulation.

Christian Effert was born in Wasserburg/Inn, Germany, in 1972. From 1992 till 1998 he studied mechanical engineering at the Technische Universitaet Muenchen. Since then he works as an assistant at the Institute for Machine Tools and Industrial Management $(i w b)$ at the Technische Universitaet Muenchen. His research interests include supply chain management, production planning and control as well as simulation. 